\title{
A Counterexample in the Theory of Local Zeta Functions
}

Roland Martin

\section{CONTENTS}

1. Introduction

2. Reductions

3. Explicit Form of $Z_{\mathbb{Q}_{p}}(s)$

4. Testing the Functional Equation

5. Remarks

Acknowledgements

References
The generalized Igusa local zeta function $Z_{\mathbb{Q}_{p}}(s)$ associated to $\left(\mathrm{SL}_{7}, \rho\right)$, where $\rho$ is the Cartan product of the first, third and fifth fundamental representations of $\mathrm{SL}_{7}$, is explicitly computed and shown not to satisfy the expected functional equation

$$
\left.Z_{\mathbb{Q}_{p}}(s)\right|_{p \mapsto p^{-1}}=p^{-7 s} Z_{\mathbb{Q}_{p}}(s) .
$$

\section{INTRODUCTION}

Igusa $[1974 ; 1975]$ inaugurated the study of local zeta functions of Igusa type. In [Igusa 1989], he introduced the generalized Igusa local zeta function and accompanied this introduction with several conjectures about the general properties of such functions. In particular, he conjectured that such functions satisfy functional equations of a certain form. This conjecture is disproved here. By way of comparison, we note that Igusa local zeta functions defined, unlike the generalized Igusa local zeta function, with respect to translation-invariant measures have been shown to satisfy functional equations of the expected form [Denef and Meuser 1991].

Let $\mathbb{Q}_{p}$ denote the field of $p$-adic numbers, where $p$ is chosen such that $\mathbb{Q}_{p}$ contains the seventh roots of unity. Let $\mathbb{Z}_{p}$ denote the ring of integers of $\mathbb{Q}_{p}$, and $p \mathbb{Z}_{p}$ the ideal of nonunits of $\mathbb{Z}_{p}$. By definition, $\mathbb{Z}_{p} / p \mathbb{Z}_{p}$ has $p$ elements. Let ||$_{p}$ be the absolute value on $\mathbb{Q}_{p}$, normalized as $|p|_{p}=p^{-1}$. Let $G^{\prime}=\mathrm{SL}_{7}$, and set $\rho=\Lambda_{1} * \Lambda_{3} * \Lambda_{5}$, the Cartan product of the first, third and fifth fundamental representations of $G^{\prime}$ as indexed by the Dynkin diagram of $G^{\prime}$. By definition, $\rho$ is the irreducible 
subrepresentation of $\Lambda_{1} \otimes \Lambda_{3} \otimes \Lambda_{5}$ containing highest weight equal the sum of the highest weights of each of $\Lambda_{1}, \Lambda_{3}$ and $\Lambda_{5}$. For ease of computation, represent $\mathrm{SL}_{7}$ in the standard basis $v_{1}, \ldots, v_{7}$ of $\mathbb{Q}_{p}^{7}$ so that $\Lambda_{3}$ has a basis of elements of the form $v_{j_{1}} \wedge \cdots \wedge v_{j_{3}}$, for $1 \leq j_{1}<\cdots<j_{3} \leq 7$, and $\Lambda_{5}$ has one of elements of the form $v_{k_{1}} \wedge \cdots \wedge v_{k_{5}}$, for $1 \leq k_{1}<\cdots<k_{5} \leq 7$.

Let $\mathbb{G}_{a}=$ Aff $^{1}$ denote the one-dimensional affine group, and $\mathbb{G}_{m}=\mathrm{GL}_{1}$ the one-dimensional linear group. The almost direct product

$$
G=\rho\left(G^{\prime}\right)\left(\mathbb{G}_{m} 1_{\operatorname{dim} \rho}\right)
$$

is a subgroup of $\mathrm{GL}_{\operatorname{dim} \rho}$ not contained in $\mathrm{SL}_{\operatorname{dim} \rho}$. Let $T$ be the maximal torus of $G$. We note here the useful fact that, with $R$ denoting the root system of $G^{\prime}$, the group $G$ is generated by $T$ and the distinguished one-dimensional subgroups $\theta_{\alpha}\left(\mathbb{G}_{a}\right)$, where $\alpha \in R$ and the homomorphisms $\theta_{\alpha}: \mathbb{G}_{a} \rightarrow G$ are such that $\mathbb{G}_{a} \cong \theta_{\alpha}\left(\mathbb{G}_{a}\right)$ and $t \theta_{\alpha}(u) t^{-1}=\theta_{\alpha}(\alpha(t) u)$ for all $t \in T$ and $u \in \mathbb{G}_{a}$. Define $f$ as the positive generator of $\operatorname{Hom}\left(G, \mathbb{G}_{m}\right)$ and $G^{0}=G_{\mathbb{Q}_{p}} \cap$ Mat $\operatorname{dim} \rho_{\operatorname{din}}\left(\mathbb{Z}_{p}\right)$, where $G_{\mathbb{Q}_{p}}$ denotes the $\mathbb{Q}_{p}$-rational points of $G$.

Definition [Igusa 1989; Martin 1996]. The generalized Igusa local zeta function associated to $\left(G^{\prime}, \rho\right)$ is

$$
Z_{\mathbb{Q}_{p}}(s)=\int_{G^{0}}|f(g)|_{p}^{s} \mu_{c}(g),
$$

where $\mu_{c}$ is Serre's canonical measure defined on $G_{\mathbb{Q}_{p}}$, and $s$ is a complex number with $\operatorname{Re} s>0$.

It is conjectured in [Igusa 1989] that $Z_{\mathbb{Q}_{p}}(s)$ has a finite form that expresses $Z_{\mathbb{Q}_{p}}(s)$ as a rational function $Z\left(p^{-1}, p^{-s}\right)$ satisfying the functional equation

$$
\left.Z\left(p^{-1}, p^{-s}\right)\right|_{p \mapsto p^{-1}}=p^{-\operatorname{deg}(f) s} Z\left(p^{-1}, p^{-s}\right) .
$$

We disprove this conjecture by finding the rational expression for $Z_{\mathbb{Q}_{p}}(s)$ and showing that (1.1) fails.

\section{REDUCTIONS}

We follow the setup of Igusa. The details are in [Martin 1992b]. Since the measure $\mu_{c}$ is difficult to work with, define a $\mathbb{Q}_{p}^{\times}\left(G_{\mathbb{Q}_{p}} \cap \mathrm{GL}_{\operatorname{dim} \rho}\left(\mathbb{Z}_{p}\right)\right)$ biinvariant function $\Phi$ on $G_{\mathbb{Q}_{p}}$ by

$$
\Phi(g)=\frac{\mu_{c}\left(g\left(G_{\mathbb{Q}_{p}} \cap \mathrm{GL}_{\operatorname{dim} \rho}\left(\mathbb{Z}_{p}\right)\right)\right)}{\mu\left(G_{\mathbb{Q}_{p}} \cap \mathrm{GL}_{\operatorname{dim} \rho}\left(\mathbb{Z}_{p}\right)\right)},
$$

where $\mu$ is the Haar measure on $G_{\mathbb{Q}_{p}}$ normalized to be canonical measure on $G_{\mathbb{Q}_{p}} \cap \mathrm{GL}_{\operatorname{dim} \rho}\left(\mathbb{Z}_{p}\right)$. It follows that $\mu_{c}(g)=\Phi(g) \mu(g)$ and, thus, that

$Z_{\mathbb{Q}_{p}}(s)=\int_{G^{0}}|f(g)|_{p}^{s+(\operatorname{dim} G / \operatorname{deg} f)} \frac{\Phi(g)}{|f(g)|_{p}^{\operatorname{dim} G / \operatorname{deg} f}} \mu(g)$.

Let $S=\left\{\alpha_{1}, \ldots, \alpha_{6}\right\}$ be the standard basis for the root system $R$. With respect to this basis, $R$ decomposes into a direct sum of positive and negative roots as $R=R^{+} \amalg R^{-}$. Define positive integers $a_{1}, \ldots, a_{6}$ by

$$
\prod_{\alpha \in R^{+}} \alpha=\prod_{i=1}^{6} \alpha_{i}^{a_{i}} .
$$

Let $\varpi$ denote the highest weight of the irreducible representation of $G$ in affine $n$-space $\mathbb{A}^{n}$ defined by $g \mapsto{ }^{t} g^{-1}$. Set $\alpha_{0}=\left.f\right|_{T}$. By construction, $\alpha_{0}, \ldots$, $\alpha_{6}$, and $\varpi$ generate $\operatorname{Hom}\left(T, \mathbb{G}_{m}\right)$ with relation

$$
\varpi^{\operatorname{deg} f}=\alpha_{0}^{-1} \prod_{i=1}^{6} \alpha_{i}^{b_{i}},
$$

defining positive integers $b_{1}, \ldots, b_{6}$.

Choose $\xi_{0}, \ldots, \xi_{6} \in \operatorname{Hom}\left(\mathbb{G}_{m}, T\right)$ satisfying the conditions $\left\langle\alpha_{i}, \xi_{j}\right\rangle=\delta_{i j}$ for $0 \leq i, j \leq 6$. It follows that $\xi_{0}, \ldots, \xi_{6}$ generate $\operatorname{Hom}\left(\mathbb{G}_{m}, T\right)$. In a common abuse of notation, the Weyl group of $G^{\prime}$ will be denoted by $\operatorname{Sym}(7)$. The action of an element $w \in$ $\operatorname{Sym}(7)$ on an element $\alpha \in R$ is given by $w(\alpha)(t)=$ $\alpha\left(w^{-1}(t)\right)$, for $t \in T$.

From the $p$-adic Bruhat decomposition and the properties of the length function $\lambda$ for the Weyl group of $\mathrm{SL}_{7}$ [Igusa 1989, pp. 703-6; Martin 1992a], we have

$$
\begin{aligned}
Z_{\mathbb{Q}_{p}}(s) & =\mu\left(G_{\mathbb{Q}_{p}} \cap \mathrm{GL}_{\operatorname{dim} \rho}\left(\mathbb{Z}_{p}\right)\right) \cdot \sum_{w \in \operatorname{Sym}(7)} p^{-\lambda(w)} \\
& \cdot\left(\sum p^{\tau\left(n_{0}, \ldots, n_{6}\right)} \frac{\Phi(\xi(\pi))}{|f(\xi(\pi))|_{p}^{\operatorname{dim} G / \operatorname{deg} f}}\right),
\end{aligned}
$$


where the summation on the second line is over all $n_{0} \in \mathbb{N}$ and all $\left(n_{1}, \ldots, n_{6}\right) \in \mathbb{N}^{6}$ such that $n_{i}>0$ if $\alpha_{i} \in w\left(R^{-}\right)$, and where

$$
\begin{gathered}
\xi=\xi_{0}^{\operatorname{deg}(f) n_{0}} \prod_{i=1}^{6}\left(\xi_{0}^{b_{i}} \xi_{i}\right)^{n_{i}} \\
\tau\left(n_{0}, \ldots, n_{6}\right)=-(49+\operatorname{deg}(f) s) n_{0} \\
+\sum_{i=1}^{6}\left(a_{i}-b_{i}\left(s+\frac{\operatorname{dim} G}{\operatorname{deg} f}\right)\right) n_{i} .
\end{gathered}
$$

\section{EXPLICIT FORM OF $Z_{\mathbb{Q}_{p}}(\mathrm{~s})$}

The explicit form for $Z_{\mathbb{Q}_{p}}(s)$ as a rational function may now be obtained. These first few computations are straightforward: $\operatorname{dim} \rho=3402, \operatorname{dim} G=$ $49, \operatorname{deg} f=7,\left(a_{1}, \ldots, a_{6}\right)=(6,10,12,12,10,6)$, and

$$
\mu\left(G_{\mathbb{Q}_{p}} \cap \mathrm{GL}_{3402}\left(\mathbb{Z}_{p}\right)\right)=\left(1-p^{-1}\right) \prod_{i=1}^{6}\left(1-p^{-(i+1)}\right) .
$$

There is also a formula for $\Phi(\xi(\pi))$, which will now be exhibited. It is a result of Élie Cartan [1913, pp. 3-5] that the weights $\omega^{*}$ of $G$ satisfy

$$
\omega^{*}=\varpi^{-1} \prod_{i=1}^{6} \alpha_{i}^{c_{i}}
$$

where $c=\left(c_{1}, \ldots, c_{6}\right) \in \Gamma$, a finite subset of $\mathbb{N}^{6}$. With these conventions, and with $n=\left(n_{1}, \ldots, n_{6}\right)$, we then have

$$
\Phi(\xi(\pi))=p^{-\left(49 n_{0}+\left(\sum_{c \in \Delta_{\xi}} c+\sum_{\alpha \in R} c_{\alpha, \xi}\right) \cdot n\right)},
$$

where $\Delta_{\xi}$ is the subset of $\Gamma$ that forms a basis for $\mathbb{Q}^{6}$ such that $c \cdot n$ for all $c \in \Delta_{\xi}$ takes the smallest value, and $c_{\alpha, \xi}$ is the $c \in \Gamma$ associated to a row $r=r_{\alpha, \xi}$ such that

$$
\left.\frac{d}{d u}\left(\theta_{\alpha}(u)\right)\right|_{u=0} \neq 0
$$

and $c \cdot n$ takes the smallest value (see [Igusa 1989, p. 713]). The proof of this fact can be found in [Martin 1992b]. Thus, the computation of $\Phi(\xi(\pi))$ involves a detailed understanding of the weights of $\rho$ and the homomorphisms $\theta_{\alpha}$, for $\alpha \in R$.

The weights of $\rho$ are easily described, and are exactly all unique products $\omega^{1} \cdot \omega^{3} \cdot \omega^{5}$, where $\omega^{1}$, $\omega^{3}$ and $\omega^{5}$ denote arbitrary weights of $\Lambda_{1}, \Lambda_{3}$ and $\Lambda_{5}$, respectively [Cartan 1913, pp. 11-13]. In particular, define $\omega_{i}: T \rightarrow \mathbb{G}_{m}$ by $\omega_{i}\left(t_{1}, \ldots, t_{7}\right)=t_{i}$, so that $\omega_{i}$ is the weight of the basis element $v_{i}$. In this notation, then,

$$
\begin{aligned}
\omega^{1}=\omega_{i} & \text { for } 1 \leq i \leq 7, \\
\omega^{3}=\omega_{j_{1}} \cdot \omega_{j_{2}} \cdot \omega_{j_{3}} & \text { for } 1 \leq j_{1}<j_{2}<j_{3} \leq 7, \\
\omega^{5}=\omega_{k_{1}} \cdots \omega_{k_{5}} & \text { for } 1 \leq k_{1}<\cdots<k_{5} \leq 7, \\
R & =\left\{\alpha_{i j}=\omega_{i} \omega_{j}^{-1}: 1 \leq i, j \leq 7 \text { and } i \neq j\right\}, \\
S & =\left\{\alpha_{i}=\omega_{i} \omega_{i+1}^{-1}: 1 \leq i \leq 6\right\} .
\end{aligned}
$$

The highest weight $\varpi$ of the irreducible representation of $G$ in $\mathbb{A}^{3402}$ defined by $g \mapsto{ }^{t} g^{-1}$ is the product $\left(\omega_{7} \cdot \omega_{5} \omega_{6} \omega_{7} \cdot \omega_{3} \ldots \omega_{7}\right)^{-1}$. It follows that $\left(b_{1}, \ldots, b_{6}\right)=(9,18,20,22,17,12)$ and that, if we set $U=\{(0,1,0,0,0,0),(0,0,0,1,0,0)$, $(0,0,0,0,0,1),(1,1,0,0,0,0)\}$, the value of $\Delta_{\xi}$ is given by the following table:

\begin{tabular}{|c|c|}
\hline condition & $\Delta_{\xi}$ \\
\hline$n_{2}<n_{4}<n_{6}$ & $U \cup\{(0,1,1,0,0,0),(0,0,0,1,1,0)\}$ \\
$n_{2}, n_{6}<n_{4}$ & $U \cup\{(0,1,1,0,0,0),(0,0,0,0,1,1)\}$ \\
$n_{2}, n_{6}>n_{4}$ & $U \cup\{(0,0,1,1,0,0),(0,0,0,1,1,0)\}$ \\
$n_{2}>n_{4}>n_{6}$ & $U \cup\{(0,0,1,1,0,0),(0,0,0,0,1,1)\}$ \\
\hline
\end{tabular}

Information about the $\theta_{\alpha_{i j}}$, for $1 \leq i, j \leq 7$ with $i \neq j$, is obtained in a more subtle fashion. Let $\theta_{\alpha_{i j}}^{\prime}\left(\mathbb{G}_{a}\right)$ denote the unipotent subgroup of $\mathrm{SL}_{7}$ composed of unipotent matrices $\theta_{\alpha_{i j}}^{\prime}(u)$ with nonidentically zero $(i, j)$-th entry $u$. We mean to exploit the fact that $\theta_{\alpha_{i j}}(u)=\rho\left(\theta_{\alpha_{i j}}^{\prime}(u)\right)$, for $u \in \mathbb{G}_{a}$. But first we consider $\Lambda_{1} \otimes \Lambda_{3} \otimes \Lambda_{5}\left(\theta_{\alpha_{i j}}^{\prime}\left(\mathbb{G}_{a}\right)\right)$, which, by construction, is defined by the action of a $\theta_{\alpha_{i j}}^{\prime}(u)$ on a basis element

$$
v_{i_{1}} \otimes v_{j_{1}} \wedge v_{j_{2}} \wedge v_{j_{3}} \otimes v_{k_{1}} \wedge \cdots \wedge v_{k_{5}} .
$$

This action is given by

$$
\begin{aligned}
& \theta_{\alpha_{i_{j}}}^{\prime}(u)\left(v_{i_{1}} \otimes v_{j_{1}} \wedge v_{j_{2}} \wedge v_{j_{3}} \otimes v_{k_{1}} \wedge \cdots \wedge v_{k_{5}}\right) \\
& =\left(\theta_{\alpha_{i j}}^{\prime}(u) v_{i_{1}}\right)\left(\theta_{\alpha_{i j}}^{\prime}(u) v_{j_{1}} \wedge v_{j_{2}} \wedge v_{j_{3}}\right)\left(\theta_{\alpha_{i_{j}}}^{\prime}(u) v_{k_{1}} \wedge \cdots \wedge v_{k_{5}}\right) .
\end{aligned}
$$


Therefore,

$$
\left.\frac{d}{d u}\left(\Lambda_{1} \otimes \Lambda_{3} \otimes \Lambda_{5}\left(\theta_{\alpha_{i j}}^{\prime}(u)\right)\right)\right|_{u=0}
$$

has nonzero entries in the rows indexed by these basis elements:

$v_{i} \otimes v_{j_{1}} \wedge v_{j_{2}} \wedge v_{j_{3}} \otimes v_{k_{1}} \wedge \cdots \wedge v_{k_{5}}$,

$v_{i_{1}} \otimes v_{l_{1}} \wedge v_{l_{2}} \wedge v_{l_{3}} \otimes v_{k_{1}} \wedge \cdots \wedge v_{k_{5}}$ for $l_{1}, l_{2}, l_{3}$

with $1 \leq l_{1}<l_{2}<l_{3} \leq 7$ and $i \in\left\{l_{1}, l_{2}, l_{3}\right\}$,

$v_{i_{1}} \otimes v_{j_{1}} \wedge v_{j_{2}} \wedge v_{j_{3}} \otimes v_{m_{1}} \wedge \cdots \wedge v_{m_{5}}$ for $m_{1}, \ldots, m_{5}$

with $1 \leq m_{1}<\cdots<m_{5} \leq 7$ and $i \in\left\{m_{1}, \ldots, m_{5}\right\}$.

Consider the case $(i, j)=(1,2)$. In this example,

$$
\left.\frac{d}{d u}\left(\Lambda_{1} \otimes \Lambda_{3} \otimes \Lambda_{5}\left(\theta_{\alpha_{12}}^{\prime}(u)\right)\right)\right|_{u=0}
$$

has nonzero entries in the rows

$v_{1} \otimes v_{j_{1}} \wedge v_{j_{2}} \wedge v_{j_{3}} \otimes v_{k_{1}} \wedge \cdots \wedge v_{k_{5}}$

$v_{i_{1}} \otimes v_{1} \wedge v_{l_{2}} \wedge v_{l_{3}} \otimes v_{k_{1}} \wedge \cdots \wedge v_{k_{5}}$

for $l_{2}, l_{3}$ with $1<l_{2}<l_{3} \leq 7$,

$v_{i_{1}} \otimes v_{j_{1}} \wedge v_{j_{2}} \wedge v_{j_{3}} \otimes v_{1} \wedge v_{m_{2}} \wedge \cdots \wedge v_{m_{5}}$

for $m_{2}, \ldots, m_{5}$ with $1<m_{2}<\cdots<m_{5} \leq 7$.

Since $v_{i_{1}} \otimes v_{j_{1}} \wedge v_{j_{2}} \wedge v_{j_{3}} \otimes v_{k_{1}} \wedge \cdots \wedge v_{k_{5}}$ has weight $\omega_{i_{1}} \cdot \omega_{j_{1}} \omega_{j_{2}} \omega_{j_{3}} \cdot \omega_{k_{1}} \cdots \omega_{k_{5}}$, and since $\varpi$ is known to be $\left(\omega_{7} \cdot \omega_{5} \omega_{6} \omega_{7} \cdot \omega_{3} \cdots \omega_{7}\right)^{-1}$, the row where

$$
\left.\frac{d}{d u}\left(\Lambda_{1} \otimes \Lambda_{3} \otimes \Lambda_{5}\left(\theta_{\alpha_{12}}^{\prime}(u)\right)\right)\right|_{u=0}
$$

has a nonzero entry and weight, with associated $c \in \Gamma$ making $c \cdot n$ minimal (where $n=\left(n_{1}, \ldots, n_{6}\right)$ ), is $v_{7} \otimes v_{5} \wedge v_{6} \wedge v_{7} \otimes v_{1} \wedge v_{4} \wedge \cdots \wedge v_{7}$. The particular $c$ here is $(1,1,0,0,0,0)$. Note that this choice for $c$ is independent of $n$, and so also independent of $\xi$. It is not always possible to arrange this, as the table on the next column will illustrate. Again from Cartan, $\rho$ has a basis of elements of the form $v=\sum_{l} c_{i, l} v_{i_{1}, l} \otimes v_{j_{1}, l} \wedge v_{j_{2}, l} \wedge v_{j_{3}, l} \otimes v_{k_{1}, l} \wedge \cdots \wedge v_{k_{5}, l}$

where $c_{i, l} \in \mathbb{Q}_{p}$ and the vectors

$$
v_{i_{1}, l} \otimes v_{j_{1}, l} \wedge v_{j_{2}, l} \wedge v_{j_{3}, l} \otimes v_{k_{1}, l} \wedge \cdots \wedge v_{k_{5}, l}
$$

are all of the same weight [Cartan 1913, pp. 1113]. This, together with the fact that the weight $\omega_{7} \cdot \omega_{5} \omega_{6} \omega_{7} \cdot \omega_{1} \omega_{4} \ldots \omega_{7}$ associated to $v_{7} \otimes v_{5} \wedge v_{6} \wedge v_{7} \otimes$ $v_{1} \wedge v_{4} \wedge \cdots \wedge v_{7}$ does occur in $\rho$ with multiplicity one and

$$
\left.\frac{d}{d u} \rho\left(\theta_{\alpha_{i j}}^{\prime}(u)\right)\right|_{u=0}
$$

has a nonzero entry in the row indexed by $v$ only if

$$
\left.\frac{d}{d u}\left(\Lambda_{1} \otimes \Lambda_{3} \otimes \Lambda_{5}\left(\theta_{\alpha_{i j}}^{\prime}(u)\right)\right)\right|_{u=0}
$$

has a nonzero entry in a row indexed by one of the $v_{i_{1}, l} \otimes v_{j_{1}, l} \wedge v_{j_{2}, l} \wedge v_{j_{3}, l} \otimes v_{k_{1}, l} \wedge \cdots \wedge v_{k_{5}, l}$, allows one

\begin{tabular}{|c|c|c|c|c|c|c|}
\hline$l$ & $j=1$ & 3 & 4 & 5 & 6 & 7 \\
\hline 1 & \multicolumn{6}{|c|}{110000110000111000111100111110111111} \\
\hline 2 & 010000 & 010000 & 011000 & 011100 & 011110 & 011111 \\
\hline 3 & 000000000000 & & $\begin{array}{l}011000 \\
001100\end{array}$ & 001100 & 001110 & 001111 \\
\hline 4 & 000000000000 & $\begin{array}{l}010000 \\
000100\end{array}$ & & 000100 & 000110 & 000111 \\
\hline 5 & 000000000000 & 000000 & 000000 & & $\begin{array}{l}000110 \\
000011\end{array}$ & 000011 \\
\hline 6 & 000000000000 & 000000 & 000000 & $\begin{array}{l}000100 \\
000001\end{array}$ & & 000001 \\
\hline 7 & 000000000000 & 000000 & 000000 & 000000 & 000000 & \\
\hline
\end{tabular}
to conclude that $v_{7} \otimes v_{5} \wedge v_{6} \wedge v_{7} \otimes v_{1} \wedge v_{4} \wedge \cdots \wedge v_{7}$ is a basis element for $\rho$. Therefore, $c_{\alpha_{12}, \xi}=c=$ $(1,1,0,0,0,0)$.

Following the same line of reasoning for the other $\alpha_{i j} \in R$, we get these values for $c_{\alpha_{i j}, \xi}$ :

We have omitted the commas and parentheses from the vectors due to typesetting limitations. Moreover, when two vectors are given for the same $(i, j)$, the correct answer depends on the $n_{i}$. Thus,

$$
\begin{aligned}
& c_{\alpha_{12}, \xi}=(1,1,0,0,0,0) \quad \text { for all } \xi, \\
& c_{\alpha_{13}, \xi}=(1,1,0,0,0,0) \quad \text { for all } \xi \text {, } \\
& c_{\alpha_{34}, \xi}= \begin{cases}(0,1,1,0,0,0) & \text { if } n_{2}<n_{4}, \\
(0,0,1,1,0,0) & \text { if } n_{2}>n_{4},\end{cases} \\
& c_{\alpha_{43}, \xi}= \begin{cases}(0,1,0,0,0,0) & \text { if } n_{2}<n_{4}, \\
(0,0,0,1,0,0) & \text { if } n_{2}>n_{4},\end{cases} \\
& c_{\alpha_{56}, \xi}= \begin{cases}(0,0,0,1,1,0) & \text { if } n_{4}<n_{6}, \\
(0,0,0,0,1,1) & \text { if } n_{4}>n_{6},\end{cases} \\
& c_{\alpha_{65}, \xi}= \begin{cases}(0,0,0,1,0,0) & \text { if } n_{4}<n_{6}, \\
(0,0,0,0,0,1) & \text { if } n_{4}>n_{6},\end{cases}
\end{aligned}
$$


and so on. The author used the Maple package crystal [Joyner and Martin 1994] to help check these computations.

The choices in $\Delta_{\xi}$ and $c_{\alpha_{i j}, \xi}$ require that the sum in parentheses in (2.1) be split into eleven pieces, reflecting the possible expressions for $\Phi(\xi(\pi))$ in terms of the relative values of $n_{2}, n_{4}$ and $n_{6}$ :

\begin{tabular}{|c|c|}
\hline condition & $\Phi(\xi(\pi))$ \\
\hline$n_{2}>n_{4}>n_{6}$ & $p^{-\left(49 n_{0}+7 n_{1}+14 n_{2}+13 n_{3}+16 n_{4}+11 n_{5}+10 n_{6}\right)}$ \\
$n_{2}>n_{4}=n_{6}$ & $p^{-\left(49 n_{0}+7 n_{1}+14 n_{2}+13 n_{3}+16 n_{4}+11 n_{5}+10 n_{6}\right)}$ \\
$n_{2}, n_{6}>n_{4}$ & $p^{-\left(49 n_{0}+7 n_{1}+14 n_{2}+13 n_{3}+19 n_{4}+11 n_{5}+7 n_{6}\right)}$ \\
$n_{2}=n_{4}>n_{6}$ & $p^{-\left(49 n_{0}+7 n_{1}+14 n_{2}+13 n_{3}+16 n_{4}+11 n_{5}+10 n_{6}\right)}$ \\
$n_{2}=n_{4}=n_{6}$ & $p^{-\left(49 n_{0}+7 n_{1}+14 n_{2}+13 n_{3}+16 n_{4}+11 n_{5}+10 n_{6}\right)}$ \\
$n_{2}=n_{4}<n_{6}$ & $p^{-\left(49 n_{0}+7 n_{1}+14 n_{2}+13 n_{3}+19 n_{4}+11 n_{5}+7 n_{6}\right)}$ \\
$n_{4}>n_{6}>n_{2}$ & $p^{-\left(49 n_{0}+7 n_{1}+17 n_{2}+13 n_{3}+13 n_{4}+11 n_{5}+10 n_{6}\right)}$ \\
$n_{4}>n_{6}=n_{2}$ & $p^{-\left(49 n_{0}+7 n_{1}+17 n_{2}+13 n_{3}+13 n_{4}+11 n_{5}+10 n_{6}\right)}$ \\
$n_{4}>n_{2}>n_{6}$ & $p^{-\left(49 n_{0}+7 n_{1}+17 n_{2}+13 n_{3}+13 n_{4}+11 n_{5}+10 n_{6}\right)}$ \\
$n_{2}<n_{4}=n_{6}$ & $p^{-\left(49 n_{0}+7 n_{1}+17 n_{2}+13 n_{3}+13 n_{4}+11 n_{5}+10 n_{6}\right)}$ \\
$n_{2}<n_{4}<n_{6}$ & $p^{-\left(49 n_{0}+7 n_{1}+17 n_{2}+13 n_{3}+16 n_{4}+11 n_{5}+7 n_{6}\right)}$ \\
\hline
\end{tabular}

As a result, we have the explicit expression for $Z_{\mathbb{Q}_{p}}(s)$ given in the sidebar below.

\section{TESTING THE FUNCTIONAL EQUATION}

It remains to check whether the functional equation holds. For notational convenience, denote by $X_{w}$ the rational function within the outer parentheses of the expression for $Z_{\mathbb{Q}_{p}}(s)$ in the sidebar.

The first step is to express $Z_{\mathbb{Q}_{p}}(s)$ with respect to the basis $\nu(S)$, where $\nu \in \operatorname{Sym}(7)$ is the unique element such that $\nu\left(R^{-}\right)=R^{+}$:

$$
\begin{aligned}
Z_{\mathbb{Q}_{p}}(s)=\frac{\left(1-p^{-1}\right) \prod_{i=1}^{6}\left(1-p^{-(i+1)}\right)}{1-p^{-(49+7 s)}} \\
\quad \cdot \sum_{w \in \operatorname{Sym}(7)} p^{-\lambda(\nu w)} \cdot \frac{p^{-Q_{\nu w}(1)(1+9 s)}}{1-p^{-(1+9 s)}} \\
\cdot \frac{p^{-Q_{\nu w}(3)(1+20 s)}}{1-p^{-(1+20 s)}} \cdot \frac{p^{-Q_{\nu w}(5)(1+17 s)}}{1-p^{-(1+17 s)}} \cdot X_{\nu w}
\end{aligned}
$$

where the $Q$-notation is defined in the sidebar. Sending $p$ to $p^{-1}$ and using the relations $\lambda(\nu w)=$ card $R^{+}-\lambda(w)$ and

$$
\left.\frac{1}{1-p^{A}}\right|_{p \mapsto p^{-1}}=\frac{-p^{A}}{1-p^{A}}
$$

\section{$Z_{\mathbb{Q}_{p}}(s)$ AS A RATIONAL FUNCTION IN $p^{-1}$ AND $p^{-s}$}

Define $Q_{w}\left(i_{1}, i_{2}, \ldots\right)$ to be equal to 1 if there exists $j \in\left\{i_{1}, i_{2}, \ldots\right\}$ such that $\alpha_{j} \in w\left(R^{-}\right)$, and equal to 0 otherwise. Then

$$
\begin{gathered}
Z_{\mathbb{Q}_{p}}(s)=\frac{\left(1-p^{-1}\right) \prod_{i=1}^{6}\left(1-p^{-(i+1)}\right)}{1-p^{-(49+7 s)}} \cdot \sum_{w \in \operatorname{Sym}(7)} p^{-\lambda(w)} \cdot \frac{p^{-Q_{w}(1)(1+9 s)}}{1-p^{-(1+9 s)}} \cdot \frac{p^{-Q_{w}(3)(1+20 s)}}{1-p^{-(1+20 s)}} \cdot \frac{p^{-Q_{w}(5)(1+17 s)}}{1-p^{-(1+17 s)}} \\
\cdot\left(\frac{p^{-(4+18 s)}}{1-p^{-(4+18 s)}}\left(\frac{p^{-(8+40 s)}}{1-p^{-(8+40 s)}} \frac{p^{-Q_{w}(6)(12+52 s)}}{1-p^{-(12+52 s)}}+\frac{p^{-Q_{w}(4,6)(12+52 s)}}{1-p^{-(12+52 s)}}+\frac{p^{-(1+12 s)}}{1-p^{-(1+12 s)}} \frac{p^{-Q_{w}(4)(12+52 s)}}{1-p^{-(12+52 s)}}\right)\right. \\
+\left(\frac{p^{-(8+40 s)}}{1-p^{-(8+40 s)}} \frac{p^{-Q_{w}(6)(12+52 s)}}{1-p^{-(12+52 s)}}+\frac{p^{-Q_{w}(2,4,6)(12+52 s)}}{1-p^{-(12+52 s)}}+\frac{p^{-(1+12 s)}}{1-p^{-(1+12 s)}} \frac{p^{-Q_{w}(2,4)(12+52 s)}}{1-p^{-(12+52 s)}}\right) \\
+\frac{p^{-(1+22 s)}}{1-p^{-(1+22 s)}}\left(\frac{p^{-(5+34 s)}}{1-p^{-(5+34 s)}} \frac{p^{-Q_{w}(6)(12+52 s)}}{1-p^{-(12+52 s)}}+\frac{p^{-Q_{w}(2,6)(12+52 s)}}{1-p^{-(12+52 s)}}+\frac{p^{-(5+34 s)}}{1-p^{-(5+34 s)}} \frac{p^{-Q_{w}(2)(12+52 s)}}{1-p^{-(12+52 s)}}\right) \\
\left.\quad+\frac{p^{-(5+34 s)}}{1-p^{-(5+34 s)}} \frac{p^{-Q_{w}(2)(12+52 s)}}{1-p^{-(12+52 s)}}+\frac{p^{-(1+12 s)}}{1-p^{-(1+12 s)}} \frac{p^{-(5+34 s)}}{1-p^{-(5+34 s)}} \frac{p^{-Q_{w}(2)(12+52 s)}}{1-p^{-(12+52 s)}}\right) .
\end{gathered}
$$


we obtain

$$
\begin{aligned}
Z_{\mathbb{Q}_{p}}(s)=p^{-7 s} \frac{\left(1-p^{-1}\right) \prod_{i=1}^{6}\left(1-p^{-(i+1)}\right)}{1-p^{-(49+7 s)}} \\
\cdot \sum_{w \in \operatorname{Sym}(7)} p^{-\lambda(w)} \cdot \frac{p^{-Q_{w}(1)(1+9 s)}}{1-p^{-(1+9 s)}} \\
\left.\cdot \frac{p^{-Q_{w}(3)(1+20 s)}}{1-p^{-(1+20 s)}} \cdot \frac{p^{-Q_{w}(5)(1+17 s)}}{1-p^{-(1+17 s)}} \cdot\left(-X_{\nu w}\right)\right|_{p \mapsto p^{-1}}
\end{aligned}
$$

Lemma. The equality $\left.Z_{\mathbb{Q}_{p}}(s)\right|_{p \mapsto p^{-1}}=p^{-7 s} Z_{\mathbb{Q}_{p}}(s)$ implies $-\left.X_{\nu}\right|_{p \mapsto p^{-1}}=X_{1}$.

Proof. Recall that $\lambda(w)=\operatorname{card}\left(R^{+} \cap w\left(R^{-}\right)\right)$. Write

$$
p^{-\lambda(w)} \frac{p^{-Q_{w}(1)(1+9 s)}}{1-p^{-(1+9 s)}} \frac{p^{-Q_{w}(3)(1+20 s)}}{1-p^{-(1+20 s)}} \frac{p^{-Q_{w}(5)(1+17 s)}}{1-p^{-(1+17 s)}} X_{w}
$$

as the Laurent series

$$
c(w){ }_{1} p^{-N(w)}+c(w){ }_{2} p^{-(N(w)+1)}+\cdots,
$$

and

$$
\begin{aligned}
p^{-\lambda(w)} \cdot \frac{p^{-Q_{w}(1)(1+9 s)}}{1-p^{-(1+9 s)}} & \cdot \frac{p^{-Q_{w}(3)(1+20 s)}}{1-p^{-(1+20 s)}} \\
& \left.\cdot \frac{p^{-Q_{w}(5)(1+17 s)}}{1-p^{-(1+17 s)}} \cdot\left(-X_{\nu w}\right)\right|_{p \mapsto p^{-1}}
\end{aligned}
$$

as

$$
c(\nu w){ }_{1} p^{-N(\nu w)}+c(\nu w)_{2} p^{-(N(\nu w)+1)}+\cdots,
$$

where the $c(w)_{i}$ and $c(\nu w)_{i}$ are constants in $\mathbb{C}$ and the $N(w)$ and $N(\nu w)$ are natural numbers. We claim that

$$
\min \{N(w): w \in \operatorname{Sym}(7)\}=N(1)=0
$$

and $N(w)>0$ if $w \neq 1$. If $w=1$, the leading term of

$p^{-\lambda(1)} \cdot \frac{p^{-Q_{1}(1)(1+9 s)}}{1-p^{-(1+9 s)}} \cdot \frac{p^{-Q_{1}(3)(1+20 s)}}{1-p^{-(1+20 s)}} \cdot \frac{p^{-Q_{1}(5)(1+17 s)}}{1-p^{-(1+17 s)}} \cdot X_{1}$

is 1 , due to the term

$$
\frac{p^{-Q_{1}(2,4,6)(12+52 s)}}{1-p^{-(12+52 s)}}=\frac{1}{1-p^{-(12+52 s)}}
$$

and the fact that $\lambda(1)=0$. Hence,

$$
c(1)_{1} p^{-N(1)}+c(1)_{2} p^{-(N(1)+1)}+\cdots
$$

equals 1 plus lower-order terms, so $N(1)=0$. Furthermore, for any other $w \in \operatorname{Sym}(7)$, we have $p^{-\lambda(w)} \neq 1$. It follows that $N(w)>N(1)$, establishing the claim. A similar argument proves that

$$
\min \{N(\nu w): w \in \operatorname{Sym}(7)\}=N(\nu)=0
$$

and $N(\nu w)>0$ if $w \neq 1$. These two results imply $-\left.X_{\nu}\right|_{p \mapsto p^{-1}}$ must equal $X_{1}$, completing the proof of the lemma.

But $-\left.X_{\nu}\right|_{p \mapsto p^{-1}}=X_{1}$ if and only if $p^{-(8+40 s)}=$ $p^{-(5+34 s)}$. Therefore, the expected functional equation $\left.Z_{\mathbb{Q}_{p}}(s)\right|_{p \mapsto p^{-1}}=p^{-7 s} Z_{\mathbb{Q}_{p}}(s)$ does not hold.

\section{REMARKS}

The function $\Phi$ is not residual in the sense of [Denef and Meuser 1991]. Further, $Z_{\mathbb{Q}_{p}}(s)$ is not simple in the sense of [Igusa 1989, p. 713], or [Martin 1996]. Thus, the fact that $Z_{\mathbb{Q}_{p}}(s)$ does not satisfy the expected functional equation does not contradict prior work. Typical questions, such as whether a modified form of the functional equation holds for $Z_{\mathbb{Q}_{p}}(s)$ and, more generally, what other representations $\rho$ have associated generalized Igusa local zeta function $Z_{\mathbb{Q}_{p}}(s)$ (for certain $p$ ) such that $Z_{\mathbb{Q}_{p}}(s)$ has a finite form that expresses $Z_{\mathbb{Q}_{p}}(s)$ as a rational function $Z\left(p^{-1}, p^{-s}\right)$ satisfying

$$
\left.Z\left(p^{-1}, p^{-s}\right)\right|_{p \mapsto p^{-1}}=p^{-\operatorname{deg}(f) s} Z\left(p^{-1}, p^{-s}\right),
$$

are being examined. Partials answers can be found in [Martin 1992a; 1996; a]. The obstruction to the expected functional equation holding for the above $Z_{\mathbb{Q}_{p}}(s)$ is due to the failure of $X_{w}=-\left.\left(X_{\nu w}\right)\right|_{p \mapsto p^{-1}}$ to hold for all $w \in \operatorname{Sym}(7)$. This failure may be traced back to the vectors

$$
\begin{aligned}
& \left(a_{1}, \ldots, a_{6}\right)=(6,10,12,12,10,6), \\
& \left(b_{1}, \ldots, b_{6}\right)=(9,18,20,22,17,12),
\end{aligned}
$$

$c \in \Delta_{\xi}$, and $c_{\alpha_{i j, \xi}}$ for $1 \leq i, j \leq 7$ and $i \neq j$. However, experimental evidence suggests that the $c_{\alpha_{i j, \xi}}$ are the key to this obstruction. Therefore, we make the following conjecture: 
Conjecture. Given a finite algebraic extension $K$ of $\mathbb{Q}_{p}$ with ring of integers $O_{K}$ and ideal of nonunits $\pi O_{K}$ satisfying

$$
\operatorname{card}\left(O_{K} / \pi O_{K}\right)=q
$$

the generalized Igusa local zeta function $Z_{K}(s)$ associated to a simply connected simple Chevalley $K$ group $G^{\prime}$ and finite dimensional $K$-representation $\rho$ of $G^{\prime}$ satisfies the expected functional equation

$$
\left.Z\left(q^{-1}, q^{-s}\right)\right|_{q \mapsto q^{-1}}=q^{-\operatorname{deg}(f) s} Z\left(q^{-1}, q^{-s}\right)
$$

if and only if the matrix of vectors $c_{\alpha_{i j, \xi}}$, where $1 \leq i, j \leq \operatorname{rank} G^{\prime}$ and $i \neq j$, has the property

$$
c_{\alpha_{i j, \xi}}=c_{\alpha_{j i, \xi}}^{\bullet}
$$

where $\left(x_{1}, x_{2}, \ldots, x_{n}\right)^{\bullet}=\left(x_{n}, x_{n-1}, \ldots, x_{1}\right)$.

Igusa has suggested that a matrix of vectors satisfying this property be called Hermitian symmetric.

\section{ACKNOWLEDGEMENTS}

I wish to thank the United States Naval Academy and, in particular, the Department of Mathematics, for technical and administrative support in the spring of 1994 during the preparation of this paper. It was there that the Maple software package crystal [Joyner and Martin 1994] was developed by David Joyner and me. The checking of the computations required in this paper was made significantly less onerous by the use of this package. I also thank Joyner for many helpful conversations and comments during the writing and editing of this paper.

\section{REFERENCES}

[Cartan 1913] Élie Cartan, "Les groupes projectifs qui ne laissent invariante aucune multiplicité plane", Bull. Soc. Math. France 41 (1913), 1-44.

[Denef and Meuser 1991] Jan Denef and Diane Meuser, "A functional equation of Igusa's local zeta function", Amer. J. Math. 113 (1991), 1135-1152.

[Igusa 1974] Jun-ichi Igusa, "Complex powers and asymptotic expansions I", J. reine angew. Math. 268/269 (1974), 110-130.

[Igusa 1975] Jun-ichi Igusa, "Complex powers and asymptotic expansions II", J. reine angew. Math. 278/279 (1975), 307-321.

[Igusa 1989] Jun-ichi Igusa, "Universal $p$-adic zeta functions and their functional equations", Amer. $J$. Math. 111 (1989), 671-716.

[Joyner and Martin 1994] David Joyner and Roland Martin, "A Maple package for the decomposition of certain tensor products of representations using crystal graphs", preprint, ftp://ftp.maplesoft.com/pub/ maple/share/5.3_combined/share/crystal.tex. The package itself can be obtained at .../crystal.

[Martin 1992a] Roland Martin, "The universal $p$-adic zeta function associated to the adjoint group of $\mathrm{SL}_{l+1}$ enlarged by the group of scalar multiples", preprint, 1992.

[Martin 1992b] Roland Martin, "On generalized Igusa local zeta functions associated to simple Chevalley $K$-groups of type $A_{l}, B_{l}, C_{l}, D_{l}, E_{6}, E_{7}, E_{8}, F_{4}$ and $G_{2}$ under the adjoint representation", preprint, 1992.

[Martin 1996] Roland Martin, "On simple Igusa local zeta functions", to appear in Electronic Research Announcements of the AMS 1 (1996), issue 3.

[Martin a] Roland Martin, "On the classification of Igusa local zeta functions associated to certain irreducible matrix groups", in preparation.

Roland Martin, Department of Mathematics, United States Naval Academy, Annapolis, MD 21402-5000

(rem@sma.usna.navy.mil)

Received March 19, 1995; accepted in revised form November 8 